\title{
Parámetros sanguíneos y perfil de hormonas reproductivas de hembras de Choloepus hoffmanni en cautiverio
}

\author{
Sofía Herra-Vargas ${ }^{1}$, Esteban Brenes-Mora ${ }^{2,5}$, Mario Baldi ${ }^{3}$, Laura Bouza-Mora ${ }^{4}$, Rose Mary \\ Huertas-Segura $^{4}$, Laura Castro-Ramírez ${ }^{1} \&$ Marcela Suárez-Esquivel ${ }^{1,3^{*}}$ \\ 1. Programa Integrado de Endocrinología y Reproducción Animal, Escuela de Medicina Veterinaria, Universidad \\ Nacional; shvrc17@gmail.com, lcastro@una.cr,msuarez@una.cr \\ 2. Escuela de Biología. Universidad de Costa Rica, Ciudad Universitaria, San José 2060, Costa Rica; \\ ebrenes18@gmail.com \\ 3. Programa de Investigación en Enfermedades Tropicales, Escuela de Medicina Veterinaria, Universidad Nacional; \\ mario.baldi.salas@una.cr \\ 4. Laboratorio de Análisis Clínicos, Escuela de Medicina Veterinaria, Universidad Nacional; laura.bouza.mora@una.cr, \\ rose.huertas.segura@una.cr \\ 5. Nai Conservation, San José 1101, Costa Rica \\ * Correspondencia
}

Recibido 25-VII-2017. Corregido 20-X-2017. Aceptado 20-XI-2017.

\begin{abstract}
Blood parameters and reproductive hormone profile of female Choloepus hoffmanni in captivity. In wild animal species, the use of non-invasive and non-stressful procedures to determine hormone profiles, such as fecal steroid measurements, has considerably increased the comprehension of their reproductive physiology. Since there is limited information related to the reproductive hormone profiles of the two-toed sloth, Choloepus hoffmani, a study was conducted in captive specimens at the "Sloth Sanctuary" (Cahuita, Limón, Costa Rica), in order to determine: (i) the reliability of the fecal progesterone and estrogen extraction and its quantification with an AIA- $360^{\circledR}$ analyzer, (ii) assess blood parameters in this species and (iii) evaluate if there is a correlation between fecal and plasmatic steroids. The study was performed over a three-month period, from November, 2013 to January, 2014, with a total amount of 208 fecal samples collected from five sexually mature females weighing $6.32 \mathrm{~kg}$ in average. The average of the median concentrations of progesterone in feces of the five females was $124.21 \mathrm{ng} / \mathrm{g}$, and $1708.95 \mathrm{pg} / \mathrm{g}$ for estrogen. The average minimal and maximal values were $50.96 \mathrm{ng} / \mathrm{g}$ and $1057.46 \mathrm{ng} / \mathrm{g}$ for progesterone and, $1191.77 \mathrm{pg} / \mathrm{g}$ and $2159.24 \mathrm{pg} / \mathrm{g}$ for estradiol. In plasma, progesterone median values were $1.26 \mathrm{ng} / \mathrm{mL}$, showing a minimum of $0.32 \mathrm{ng} / \mathrm{mL}$ and $12.84 \mathrm{ng} / \mathrm{mL}$ as maximum values. The plasmatic estrogen levels were below the detection limit of the equipment $(25 \mathrm{pg} / \mathrm{mL})$. Although there was no strong statistical correlation between the fecal and plasmatic progesterone fluctuations, our data suggests that the plasmatic events are mostly reflected in feces two days afterwards. Also, the levels of progesterone were elevated during the first half of November and, subsequently, showed a successive and important reduction in all the females tested. Finally, our results demonstrated that fecal steroid extractions and their measurement in a AIA-360 ${ }^{\circledR}$, allowed the successful detection and represents an alternative non-invasive determination of hormone profiles in C. hoffmani. Rev. Biol. Trop. 66(1): 280-292. Epub 2018 March 01.
\end{abstract}

Key words: progesterone; estrogen; feces; estrous cycle; hematology; sloths; AIA-360.

La obtención de información fisiológica a partir de poblaciones de animales silvestres en cautiverio es un recurso que puede ayudar a entender muchas de las características reproductivas de una especie en vida libre, esto en aras de su conservación, en especial para especies silvestres amenazadas (Paris, Whiteb,
Reissb, Westa, \& Schwarzenberger, 2002; Graham, 2004; Dias, dos-Santos, Lara, Righetto, Pinder, \& Chiarello, 2007). Para ello, es imprescindible conocer los parámetros reproductivos básicos de cada especie, incluyendo las características del ciclo estral, la edad del inicio de la pubertad, y el tiempo de gestación entre otras 
variables (Graham, Schwarzenberger, Möstl, Galama, \& Savage, 2001; Palme, 2005; Pereira, Barbanti, \& Negrão, 2005; Touma \& Palme, 2005; Dumonceaux, Bauman, \& Carillo, 2006; Dias, et al., 2007; Schwarzenberger, 2007). Por lo tanto, es necesario conocer los patrones endocrinos de cada especie, información fundamental para entender su biología reproductiva (Graham, 2004).

Tradicionalmente, los perfiles endocrinos han sido determinados con base en las fluctuaciones hormonales en sangre (Goymann, 2012; Palme, 2005). Sin embargo, por las implicaciones de bienestar animal, complicaciones asociadas con la manipulación de los animales y la facilidad de incrementar el tamaño de la muestra, se ha buscado la implementación de métodos no invasivos para la determinación de los diferentes estados fisiológicos, reduciendo con ello, los efectos negativos de estas metodologías de tipo invasivas (Valdespino, Martínez-Mota, García-Feria, \& Martínez-Romero, 2007; Goymann, 2012). Por esta razón, las técnicas no invasivas utilizadas para establecer los niveles de esteroides en heces han experimentado un mejora sustancial en las últimas décadas (Graham et al., 2001; Goymann, 2012; Keay, Singh, Gaunt, \& Kaur, 2006; Schwarzenberger, 2007).

Dentro de las ventajas de las técnicas no invasivas, sobresale el hecho de estar menos influenciadas por factores como el estrés por la manipulación, se facilita la recolección de muestras seriadas por animal con una mínima interacción sobre el individuo, facilitando estudios paralelos del comportamiento reproductivo. Además, descarta el inconveniente de no obtener una cantidad adecuada de muestra, contrario a lo que ocurre por ejemplo con muestras de sangre (Chelini, Souza, Rocha, Felippe, \& Oliveira, 2005; Goymann, 2005; Dumonceaux et al., 2006; Schwarzenberger, 2007).

El método de análisis de esteroides en heces se fundamenta en el principio de detección de compuestos hormonales de "desecho" producto de la metabolización. Los metabolitos conservan su estructura esteroidea, permitiendo su detección y cuantificación en heces, facilitando el establecimiento de relaciones entre las variaciones de esteroides en sangre y su eliminación en las heces (Touma \& Palme, 2005).

Si bien, el perezoso de dos dedos (Choloepus hoffmanni) es uno de los mamíferos más abundantes en las zonas boscosas de Costa Rica (Wainwright, 2007), existen pocos estudios que contemplan aspectos reproductivos y endocrinos de esta u otras especies del super-orden Xenarthra (Gilmore, Da-Costa, \& Duarte, 2000; Mühlbauer, Duarte, Gilmore, \& Da-Costa, 2006; Troll et al., 2013). Snoek, Cruz, Catenacci y Cassano (2011), atribuyen esto al comportamiento críptico del C. hoffmanni, el cual los hace difíciles de estudiar en condiciones de vida libre. Además, la literatura reporta que las hembras de C. hoffmanni carecen de características sexuales secundarias o una clara evidencia de estro, siendo su manifestación muy sutil, con leve edematización de la vulva y escasa secreción vaginal (Gilmore et al., 2000). Esto hace que la determinación del celo por métodos observacionales en condiciones de campo, sea difícil o imposible.

Distintos reportes coinciden en que las hembras alcanzan su madurez sexual a la edad de dos años, mientras que los machos a los cuatro años (Taube, Keravec, Vié, \& Duplantier, 2001; Wainwright, 2007). Se considera que la reproducción de $C$. hoffmanni en vida libre sigue un patrón de estacionalidad, siendo más comunes las gestaciones durante la época lluviosa y los partos durante los meses más secos (Taube et al., 2001). La preñez en esta especie tiene una duración que ronda los 11 meses, con un periodo de lactancia de un mes. Las crías acompañan a la madre hasta los cinco meses de edad.

En la literatura se hace referencia al uso de métodos tradicionales en otras especies para conocer el estado hormonal, tales como la citología vaginal, la colecta de orina y la toma de muestras sanguíneas, los cuales son logísticamente complicados y laboriosos, en especial para la especie C. hoffmanni dada su conducta agresiva (Snoeck et al., 2011; Troll et al., 2013). Por tanto, el análisis de metabolitos 
hormonales en heces representa un método de medición alternativo para el monitoreo reproductivo de esta especie a largo plazo.

El objetivo de este estudio fue realizar un seguimiento del ciclo reproductivo en $C$. hoffmanni en condiciones de cautiverio, como modelo para conocer la biología reproductiva de esta especie y de esa manera, valorar la utilidad de las extracciones de metabolitos hormonales fecales en nuestras condiciones.

\section{MATERIALES Y MÉTODOS}

Selección del sitio de muestreo y de los animales: El muestreo se llevó a cabo en "Sloth Sanctuary" (9॰47'57" - 9०47'58”N \& 8254'51” - 8254'54”W) en Cahuita, Limón, Costa Rica, durante los meses de noviembre y diciembre de 2013 y enero de 2014. Fueron seleccionadas 19 hembras sexualmente maduras de C. hoffmanni, con edades estimadas entre cuatro y 12 años, peso promedio de 6.75 $\mathrm{kg}(5 \mathrm{~kg}-8.47 \mathrm{~kg})$ y aparentemente sanas, sin historia de padecimientos recientes acorde con los expedientes clínicos de cada animal. Estos individuos cumplieron con los siguientes criterios de inclusión: (i) los animales debían estar bajo las mismas condiciones de temperatura, ubicación y tamaño del recinto; (ii) tener el mismo manejo nutricional; y (iii) cada sujeto debía estar alojado en un recinto individual.

La dieta establecida para todos los individuos incluyó vainicas (Phaseollus vulgaris), chayote (Sechium edule), zanahoria (Daucus carota) y berros chinos (Nasturtium officinale).

Los recintos miden $2 \mathrm{~m}$ de ancho, $3 \mathrm{~m}$ de fondo y $2 \mathrm{~m}$ de alto. Se encuentran distribuidos uno junto al otro en un espacio de acceso limitado del refugio. Son de cemento y poseen paredes de malla, de manera que los animales están en constante contacto visual y olfatorio entre sí; dentro de los recintos hay una estructura metálica, así como una tarima y ramas para que se desplacen.

Todos los permisos de esta investigación fueron aprobados por el Comité de Bioética y Bienestar Animal de la Escuela de Medicina Veterinaria, Universidad Nacional
(FCSA-EMV-CBAB-007-2014) y se contó con el consentimiento de la administración del Sloth Sanctuary.

Muestras de sangre: Fueron tomadas directamente de la vena cubital o de la vena subclavia en todas las hembras estudiadas, previa sedación de los animales con una combinación intramuscular de Ketamina-Medetomidina (2.5 $\mathrm{mg} / \mathrm{kg}+0.02 \mathrm{mg} / \mathrm{kg}$ respectivamente) (Domitor $^{\circledR}$; Zoetis Kalamazoo, MI 49007, USA.Ketamine; Aveco Co., Fort Dodge, IA, USA). Luego de 45 minutos de anestesia se antagonizó la Medetomidina con una dosis de Atipamazole $0.1 \mathrm{mg} / \mathrm{kg}$ IM (Antisedan ${ }^{\circledR}$; Zoetis, Kalamazoo, MI 49007) (Hanley, 2008).

Un volumen $\sim 1.5 \mathrm{~mL}$ de sangre fue obtenido de cada animal usando una jeringa heparinizada (heparina de litio) y cada muestra fue divida en dos alícuotas, una se almacenó en un tubo con heparina $(\sim 500 \mu \mathrm{L})$ y la segunda muestra fue reservada para colectar plasma siguiendo el procedimiento estándar. Las alícuotas se transportaron a $\sim{ }^{\circ} \mathrm{C}$ en una hielera en menos de 24 horas para su posterior análisis hematológico y de química sanguínea, en el Laboratorio de Análisis Clínicos de la Escuela de Medicina Veterinaria de la Universidad Nacional (EMV), donde se mantuvieron a $4^{\circ} \mathrm{C}$ hasta su procesamiento. Las muestras sanguíneas de cada perezoso seleccionado (Cuadro 1), se tomaron con una frecuencia de 15 días durante un período de tres meses (noviembre 2013 a enero de 2014).

\section{Análisis hematológico, química sanguí-}

nea: Cada muestra de sangre completa y/o plasma, fue procesada en un periodo no mayor a las 24 a 48 horas posteriores a su recolección. Se evaluó el hemograma completo, y se determinó la concentración de proteínas en plasma (totales y albúmina). Adicionalmente, se analizaron los metabolitos de nitrógeno ureico, creatinina y las enzimas: Aspartato Aminotransferasa (AST) y Alanina Aminotransferasa (ALT). El análisis hematológico se llevó a cabo siguiendo el procedimiento estándar establecido por Meneses-Guevara y Bouza-Mora 
CUADRO 1

Edades, número total de muestras de heces analizadas para cuantificación hormonal y pesos de las hembras incluidas en el estudio hormonal.

TABLE 1

Ages, total number of fecal samples analyzed for hormonal quantification and weights of females included in hormonal study

\begin{tabular}{lccccccccc} 
& & \multicolumn{9}{c}{ Pesos por visita (kg) } \\
Identificación & Edad estimada (años) & $\mathrm{n}$ & \multicolumn{9}{c}{2013} & \multicolumn{2}{c}{2014} \\
& & & 14.11 & 28.11 & 12.12 & 28.12 & 13.01 & 28.01 \\
Hembra 1 & 5 & 42 & 5.6 & 5.7 & 5.9 & 6.0 & 5.8 & 6.1 \\
Hembra 2 & 11 & 55 & 7.2 & 7.3 & 7.7 & 8.1 & 7.9 & 8.3 \\
Hembra 3 & 11 & 33 & 7.9 & 7.4 & 7.5 & 7.3 & 7.7 & 7.3 \\
Hembra 4 & 4 & 46 & 5.2 & 5.5 & 5.3 & 5.4 & 5.6 & 5.7 \\
Hembra 5 & 12 & 35 & 5.1 & 5.0 & 4.9 & 4.8 & 5.2 & 5.2 \\
\hline
\end{tabular}

(2014), el cual básicamente consisten en un montaje manual del hematocrito, hemoglobina y conteo leucocitario, así como la realización del diferencial celular bajo la observación de personal capacitado y con amplia experiencia, esto con el fin de evitar errores de lectura en los equipos automatizados. La química sanguínea se cuantificó mediante el equipo automatizado Selectra Junior Spinlab100® (Vital Scientific, Van Rensselaerweg 4, AV Spankeren, NL 6956, USA) siguiendo las recomendaciones del fabricante (Meneses-Guevara \& Bouza-Mora, 2014).

Material fecal: Muestras de heces de todas las hembras seleccionadas fueron recolectadas cada vez que defecaron, usando para ello un guante de látex estéril nuevo para cada muestra. La identificación de las muestras se garantizó al mantener una sola hembra por recinto. Las heces se almacenaron en bolsas estériles con la identificaron del individuo incluyendo la fecha de recolección. Todas las muestras fueron almacenadas inmediatamente a $-20{ }^{\circ} \mathrm{C}$ y se procesaron en los tres meses posteriores a su colecta en el Laboratorio de Endocrinología y Biotecnología Reproductiva de la EMV. Se decidió incluir en esta parte del estudio a solo cinco hembras, las cuales poseyeron el mayor número de heces y mayor confiabilidad en la frecuencia cronológica de defecaciones; esto permitió una colecta de muestras de manera sistemática. En total, se analizaron 208 muestras: $42,55,33,43$ y 35 de cada uno de los individuos, respectivamente (Cuadro 1).

\section{Extracción de metabolitos hormonales} en heces: Se fundamentó en el método de Brown, Walker y Steinman (2009) para muestras de heces húmedas. En el cual a partir de $0.5 \mathrm{~g}$ de heces se extrajeron metabolitos hormonales usando etanol al $90 \%$. Este material se colocó en un Thermomixer ${ }^{\circledR} \mathrm{C}$ (Eppendorf®, Hamburg, Germany) a $96{ }^{\circ} \mathrm{C}$ durante 20 minutos; seguidamente se centrifugó a $2500 \mathrm{rpm}$ durante 20 minutos, se recuperó el sobrenadante. Se conservó el sobrenadante y al precipitado remanente se le repitió el procedimiento anterior. Finalmente, se combinaron los dos sobrenadantes en una sola solución y se evaporó a $56^{\circ} \mathrm{C}$ por 60 minutos, bajo un flujo de aire comprimido. Posteriormente, se le agregó $1 \mathrm{~mL}$ de solución salina estéril y se homogenizó. A partir de esta solución, se realizaron diluciones seriadas (1:3) hasta llegar a una relación final de 1:81; esta última solución se empleó inmediatamente para la cuantificación de hormonas. Los remanentes de las diluciones fueron almacenados a $-20{ }^{\circ} \mathrm{C}$. Previo a las extracciones en las muestras de las hembras seleccionadas, se estableció la eficiencia del método de extracción y la repetitividad de las mediciones hormonales en heces (material suplementario S1), según lo establecido por Brown et al. (2009). 
Cuantificación hormonal: Las mediciones hormonales en sangre de progesterona $\left(\mathrm{P}_{4}\right)$ $\mathrm{y}$ estradiol $\left(\mathrm{E}_{2}\right)$, al igual que los metabolitos hormonales en heces, se cuantificaron utilizando un equipo automatizado (Tosoh Bioscience, Inc. Modelo AIA-360 ${ }^{\circledR}$. 6000 Shoreline Court, Suite 101 South San Francisco, CA. USA) siguiendo las recomendaciones del fabricante para ambos casos. Brevemente, este equipo realiza una detección hormonal o de metabolitos hormonales por medio de un ensayo de tipo competitivo basado en la inmunoabsorción ligado a enzimas fluorescentes (ELFIA), mediante las copas comerciales ST AIA PACK PROG ${ }^{\circledR}$ y ST AIA PACK E2 ${ }^{\circledR}$ marca Tosoh Bioscience. Las copas empleadas contienen "perlas" magnetizadas impregnadas con anticuerpos para las hormonas o metabolitos específicos, y se usan enzimas marcadas con un antígeno de interés (hormonas o metabolitos específicos) para realizar la detección y cuantificación. La cuantificación se realizó con la medición de la cantidad de enzima que se une a las perlas, la cual es inversamente proporcional a la concentración de hormona en la muestra. Para este ensayo se usaron $75 \mu \mathrm{L}$ de muestra de plasma o $5 \mu \mathrm{L}$ de extracto de metabolitos de heces.

De acuerdo con los datos suministrados por el fabricante, ambos kits comerciales muestran un $100 \%$ de especificidad con la hormona correspondiente $\left(\mathrm{P}_{4} \mathrm{o} \mathrm{E}_{2}\right)$. Se reportan porcentajes de reacción cruzada con otros esteroides inferiores a $6.9 \%$ para las copas ST AIA PACK PROG $^{\circledR}$ y $7.2 \%$ para las copas ST AIA PACK E2 ${ }^{\circledR}$ (material suplementario S1). Los rangos de detección son 0.1 a $40 \mathrm{ng} / \mathrm{dL}$ y 25 a 3000 $\mathrm{pg} / \mathrm{mL}$, respectivamente.

Comportamiento del ciclo estral: Para estimar la duración del ciclo estral, por medio de la actividad ovárica en los animales seleccionados, se siguió la metodología de Troll et al. (2013), donde a partir de la concentración máxima y mínima de metabolitos de $\mathrm{P}_{4}$ en heces $\left(\mathrm{P}_{4} \mathrm{H}\right)$ durante el tiempo, se estimó la actividad ovárica y con ello la duración del ciclo estral. A partir de estos datos se definieron dos grupos de valores bajo los siguientes criterios: "altos" aquellos datos de $\mathrm{P}_{4} \mathrm{H}$ con valores $>1.5$ veces a su desviación estándar (DS) del promedio del metabolito de $\mathrm{P}_{4}$ de cada animal; y "medios" aquellos valores $<1.5$ a su DS. Se consideró que cada valor "alto" representó un pico de $\mathrm{P}_{4}$ en plasma y por tanto, el tiempo transcurrido entre picos, sugirió la duración del ciclo estral.

Para determinar las concentraciones hormonales por gramo de heces de $\mathrm{P}_{4}(\mathrm{ng} / \mathrm{g})$ y de $\mathrm{E}_{2}(\mathrm{pg} / \mathrm{g})$, se calcularon los valores mínimos y máximos, mediana, promedios y la diferencia promedio entre hembras. Estas diferencias se analizaron descriptivamente y se determinó su relevancia por criterios veterinarios.

Adicionalmente, se correlacionó la concentración hormonal entre plasma y sus metabolitos hormonales fecales mediante la prueba no paramétrica de Spearman. Para esto se consideraron las muestras de heces del mismo día del sangrado y los datos obtenidos en los cuatro días siguientes, al considerar la duración del tránsito intestinal.

Los análisis estadísticos se efectuaron con la plataforma R 3.1.2 (R Core Team, 2014) utilizando los paquetes "multicon" (Sherman, 2015), "descTools" (Signorell et al., 2017) y "ggplot2" (Wickham, 2009).

\section{RESULTADOS}

Observaciones generales: Las hembras seleccionadas para el seguimiento hormonal presentaron un promedio de 3.5 deposiciones con un rango de una a cinco por semana, esto representó un elevado número de muestras de heces $(n=208)$ durante los meses del muestreo (Cuadro 1). Durante las visitas no se detectaron signos de celo y los cuidadores reportaron ausencia de cambios de comportamiento compatibles con estro. Se descartó la posibilidad de gestación durante el periodo de seguimiento, ya que las hembras se encontraban en recintos individuales. A lo largo del estudio las hembras mostaron variaciones leves en su peso (Cuadro 1, material suplementario S2); no se observaron otros hallazgos que indicaran alteraciones en el estado de salud que pudieran afectar los resultados obtenidos. 
Hematología, química clínica y hormonas en plasma: Se analizaron un total de 56 muestras sanguíneas pertenecientes a 19 hembras C. hoffmanni, las cuales mostraron un elevado recuento plaquetario y un leucograma compuesto principalmente por linfocitos (Cuadro 2, material suplementario S2).

Las cinco hembras seleccionadas para el seguimiento reproductivo mostraron concentraciones de $\mathrm{P}_{4}$ plasmática con una mediana de $1.26 \mathrm{ng} / \mathrm{mL}$, con un mínimo de $0.3 \mathrm{ng} / \mathrm{mL}$ y $12.84 \mathrm{ng} / \mathrm{mL}$ como valor máximo; los resultados de $\mathrm{E}_{2}$ se encontraron por debajo del límite de detección del equipo empleado (Cuadro 3, material suplementario S2).

Metabolitos hormonales en heces: El método de extracción en nuestras condiciones mostró un promedio de recuperación hormonal de $83 \%$, con una variación intra-ensayo de $13.8 \%$ para la progesterona y $9.4 \%$ para el estradiol (material suplementario S1). Las cinco hembras seleccionadas defecaron con una frecuencia de una a cinco deposiciones semanales, con un promedio general de 3.55 (IC 95 \%: 3.36 - 3.74) veces por semana. El promedio de las concentraciones medianas en las heces de las cinco hembras fue de 124.21 ng/g para la $\mathrm{P}_{4}$ y $1708.95 \mathrm{pg} / \mathrm{g}$ para $\mathrm{E}_{2}$. Las concentraciones promedio mínimas y máximas fueron $50.96 \mathrm{ng} / \mathrm{g}$ y $1057.46 \mathrm{ng} / \mathrm{g}$ para $\mathrm{P}_{4}$ y para $\mathrm{E}_{2}, 1191.77 \mathrm{pg} / \mathrm{g}$ y $2159.24 \mathrm{pg} / \mathrm{g}$ (Cuadro 3 y Fig. 1). El detalle de los resultados por hembra se detalla en el material suplementario S3.

La "Hembra 5" presentó un promedio de $\mathrm{P}_{4} \mathrm{H}$ inferior al observado en el resto de los individuos. De igual manera la concentración promedio de $\mathrm{E}_{2}$ en heces $\left(\mathrm{E}_{2} \mathrm{H}\right)$ fue muy

CUADRO 2

Promedio y su IC 95\%, mediana, valores mínimos y máximos de los resultados de hematología y química sanguínea en 19 hembras $C$. hoffmanni

TABLE 2

Average and CI 95\%, median, minimal and maximal values of hematological and biochemical results in 19 C. hoffmanni females

\begin{tabular}{|c|c|c|c|}
\hline Variable & $\mathrm{n}$ & Promedio \pm IC $95 \%$ & Mediana (Min-Max) \\
\hline Hematocrito (\%) & 52 & $35 \pm 1$ & $35.16(29-40)$ \\
\hline Hemoglobina (g/dl) & 56 & $10.95 \pm 0.24$ & $10.87(9.4-13)$ \\
\hline $\mathrm{HCM}^{*}(\mathrm{~g} / \mathrm{dL})$ & 52 & $31 \pm 1$ & $31.69(27-37)$ \\
\hline Conteo de leucocitos $(\mu \mathrm{L})$ & 56 & $12502 \pm 1242$ & $12577(4150-24500)$ \\
\hline N. $\$$ en banda $(\mu \mathrm{L})$ & 56 & $0 \pm 0$ & $0(0-0)$ \\
\hline N. ${ }^{\ddagger}$ segmentados $(\mu \mathrm{L})$ & 56 & $3123 \pm 649$ & $2220(124-14740)$ \\
\hline Eosinófilos $(\mu \mathrm{L})$ & 56 & $491 \pm 429$ & $303(0-5975)$ \\
\hline Basófilos $(\mu \mathrm{L})$ & 56 & $115 \pm 52$ & $0(0-882)$ \\
\hline Linfocitos $(\mu \mathrm{L})$ & 56 & $8764 \pm 1065$ & $8335(2040-15200)$ \\
\hline Monocitos $(\mu \mathrm{L})$ & 56 & $0 \pm 0$ & $0(0-0)$ \\
\hline Plaquetas $(\mu \mathrm{L})$ & 52 & $173130 \pm 100677$ & $99824(25690$ - 2003382$)$ \\
\hline Proteínas totales (g/dL) & 54 & $8.30 \pm 3.34$ & $6.85(3.9-66.6)$ \\
\hline Albúmina (g/dL) & 55 & $3.82 \pm 0.24$ & $3.90(2-4.9)$ \\
\hline Nitrógeno uréico (mg/dL) & 55 & $18.63 \pm 1.56$ & $19(9-28)$ \\
\hline Creatinina (mg/dL) & 50 & $0.83 \pm 0.08$ & $0.90(0.4-1.2)$ \\
\hline $\mathrm{AST}^{\dagger}(\mathrm{U} / \mathrm{L})$ & 56 & $94 \pm 9$ & $95(52-149)$ \\
\hline $\operatorname{ALT}^{\S}(\mathrm{U} / \mathrm{L})$ & 56 & $8 \pm 2$ & $8(1-29)$ \\
\hline
\end{tabular}

* Hemoglobina corpuscular media.

+ Neutrófilos.

$\dagger$ Aspartato amino transferasa.

$\S$ Alanina amino transferasa. 
CUADRO 3

Promedio y su IC95\%, mediana, valores mínimos y máximos de los resultados de hormonas en heces y plasma de las cinco hembras seleccionadas para seguimiento reproductivo

TABLE 3

Average and CI $95 \%$, median, minimal and maximal values of hormones in feces and plasma of the selected females for reproductive follow up

\begin{tabular}{|c|c|c|c|}
\hline \multicolumn{2}{|c|}{ Variable } & Promedio \pm IC $95 \%$ & Mediana (Min-Max) \\
\hline \multicolumn{2}{|c|}{$\mathrm{P}_{4}$ plasmática $(\mathrm{ng} / \mathrm{mL})^{+}$} & $3.11 \pm 0.92$ & $1.26(0.32-12.84)$ \\
\hline \multicolumn{2}{|c|}{$\mathrm{E}_{2}$ plasmático $(\mathrm{pg} / \mathrm{mL})^{+}$} & $<25^{*}$ & $<25^{*}$ \\
\hline \multirow[t]{6}{*}{$\mathrm{P}_{4}$ en heces $(\mathrm{ng} / \mathrm{g})$} & Hembra 1 & $188.19 \pm 58.44$ & $94.22(50.74-838.85)$ \\
\hline & Hembra 2 & $216.09 \pm 62.86$ & $90.79(42.72-999.83)$ \\
\hline & Hembra 3 & $264.62 \pm 85,60$ & $196.46(52.26-1048.28)$ \\
\hline & Hembra 4 & $297.49 \pm 99.24$ & $177.38(62.56-1526.00)$ \\
\hline & Hembra 5 & $120.90 \pm 53.49$ & $62.18(46.54-874.33)$ \\
\hline & Hembras $1-5^{+}$ & $217.46 \pm 81.15$ & $124.21(50.96-1057.46)$ \\
\hline \multirow[t]{6}{*}{$E_{2}$ en heces $(\mathrm{pg} / \mathrm{g})$} & Hembra 1 & $1767.22 \pm 52.31$ & $1756.48(1469.66$ - 2339.96$)$ \\
\hline & Hembra 2 & $1772.65 \pm 50.85$ & $1733.02(1472.69-2$ 211.30) \\
\hline & Hembra 3 & $1719.30 \pm 72.20$ & $1787.51(1269.87$ - 2 197.68) \\
\hline & Hembra 4 & $1560.44 \pm 71.7$ & $1549.88(239.14-1902.54)$ \\
\hline & Hembra 5 & $1700.61 \pm 46.09$ & $1717.88(1507.50-2144.71)$ \\
\hline & Hembras $1-5^{+}$ & $1704.04 \pm 106.75$ & $1708.95(1191.77$ - 2 159.24) \\
\hline
\end{tabular}

+ Valores promedio considerando las 5 hembras a las que se realizó seguimiento reproductivo.

* Valores inferiores al límite inferior de detección del equipo AIA-360 ${ }^{\circledR}$.

similar en cuatro de las hembras (KruskalWallis $=0.932, \mathrm{P}=0.818$ ), con excepción de la "Hembra 4", quien presentó valores inferiores a $1600 \mathrm{pg} / \mathrm{g}$ (Kruskal-Wallis $=34.709$, $\mathrm{P}=5.33 \times 10^{-7}$ ).

Comportamiento del ciclo estral: El cambio de las concentraciones de los metabolitos de $\mathrm{P}_{4} \mathrm{H}$ y $\mathrm{E}_{2} \mathrm{H}$ de cinco hembras se siguió durante tres meses (Fig. 2). Se observó un pico de $\mathrm{P}_{4} \mathrm{H}$ en todas las hembras a inicios de noviembre, momento a partir del cual decayó progresivamente la actividad progestacional hasta alcanzar valores inferiores a $100 \mathrm{ng} / \mathrm{g}$ con leves variaciones (Fig. 2). Asimismo, se detectó la presencia de fluctuaciones irregulares del metabolito de $\mathrm{E}_{2} \mathrm{H}$ durante todo el periodo de análisis. Solamente dos hembras (hembras uno y dos) presentaron alta regularidad en su ciclo durante el periodo de muestreo (Fig. 3).
A

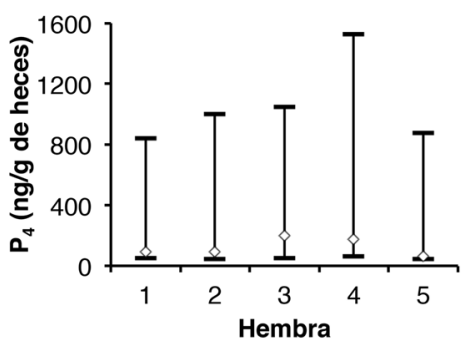

B

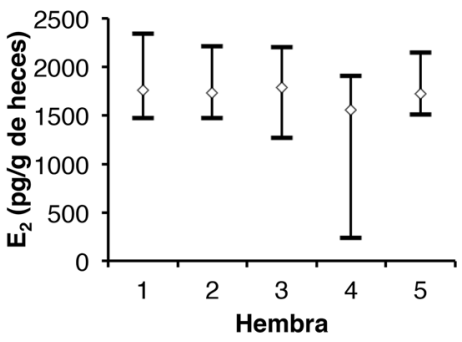

Fig. 1. Mediana, valores mínimos y máximos de hormonas en heces de las cinco hembras estudiadas. A. Resultados de progesterona. B. Resultados de estradiol.

Fig. 1. Median, minimal and maximal values of fecal hormones in the five studied females. A. Progesterone results. B. Estradiol results. 


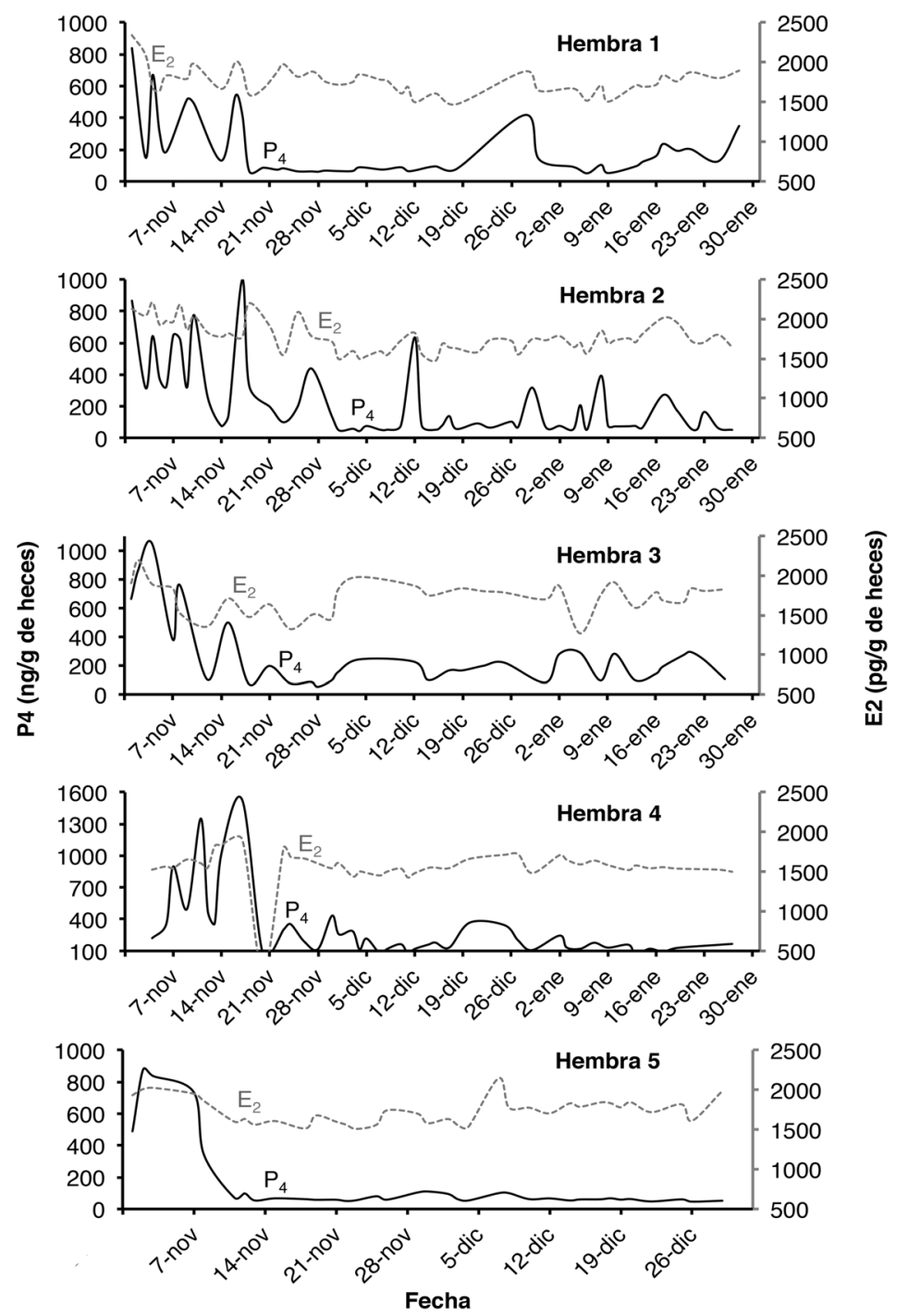

Fig. 2. Concentración de $\mathrm{P}_{4}$ (ng/g de heces) y $\mathrm{E}_{2}$ (pg/g de heces) de cinco hembras durante tres meses (noviembre y diciembre de 2013 y enero de 2014). La línea sólida representa la $\mathrm{P}_{4}$, la línea punteada corresponde al $\mathrm{E}_{2}$.

Fig. 2. $P_{4}$ (ng/g of feces) and $E_{2}$ (pg/g of feces) concentrations in feces during three months (November - December 2013 and January 2014). Solid lines represents $\mathrm{P}_{4}$ and dotted lines corresponds to $\mathrm{E}_{2}$.

Correlación entre la concentración hormonal de progesterona en sangre y en heces: No se encontró una correlación estadísticamente significativa entre la progesterona plasmática y la $\mathrm{P}_{4} \mathrm{H}$ en ninguno de los días evaluados (Spearman, P > 0.05; Cuadro 4).

\section{DISCUSIÓN}

Los valores hematológicos y de química sanguínea encontrados en este estudio concuerdan con los rangos y proporciones reportados por otros autores para la especie C. hoffmanni 


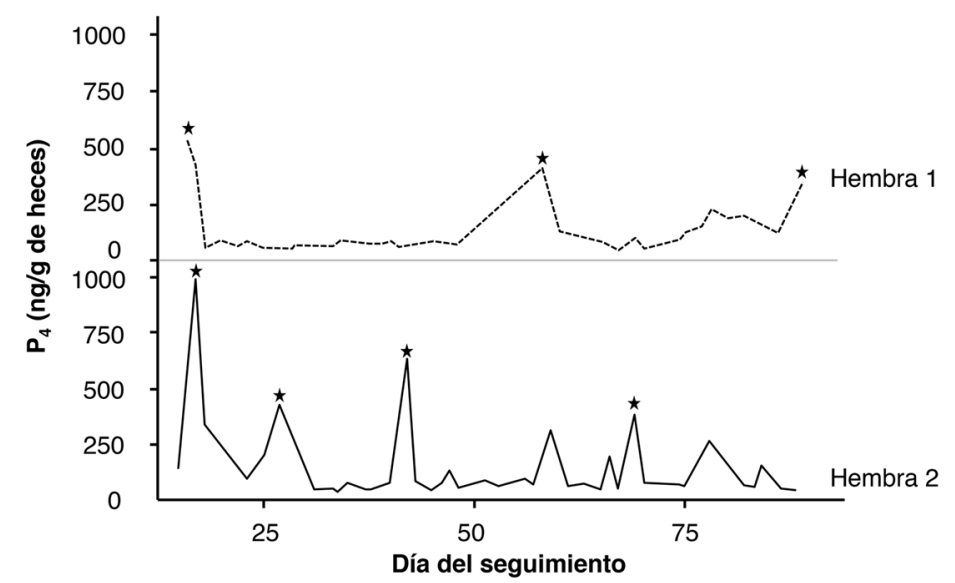

Fig. 3. Estimación del ciclo estral en las hembras uno y dos durante noviembre y diciembre de 2013 y enero de 2014. Las estrellas negras indican los momentos en los que ocurrieron picos de $\mathrm{P}_{4} \mathrm{H}$.

Fig. 3. Estral cycle estimation in females 1 and 2, on November - December 2013 and January 2014. Black stars indicate fecal progesterone increases.

\section{CUADRO 4}

Correlación (r) y su IC $95 \%$ entre las muestras de sangre y las heces del mismo día de la toma sanguínea (día 0) y los cuatro días posteriores

TABLE 4

Correlation (r) and CI $95 \%$ between blood samples and feces collected from the blood sampling day (day 0) and the four next days.

\begin{tabular}{cccc} 
Día & $\mathrm{n}$ & $\mathrm{r}$ & $\mathrm{IC} 95 \%$ \\
0 & 10 & -0.29 & $-0.78-0.42$ \\
1 & 6 & -0.024 & $-0.82-0.80$ \\
2 & 8 & 0.81 & $0.24-0.96$ \\
3 & 10 & 0.21 & $-0.48-0.74$ \\
4 & 5 & 0.34 & $-0.77-0.94$ \\
\hline
\end{tabular}

(Durán, 2005; Hayssen, 2011; Kinney, Cole, Vaughan, \& Sladky, 2013; Miller \& Fowler, 2015); de igual manera, hay concordancia de los resultados hematológicos con lo reportado para las especies Choloepus didactylus (Vogel, Vie, Thoisy, \& Moreau, 1999) y Bradypus variegatus (Araújo, 2006). Aunque C. didactylus no es la misma especie de este estudio, sí es del mismo género Choloepus, por lo que representa una especie simpátrica para el hemisferio sur y por tanto, los valores en nuestro estudio presentan comportamientos similares. Reportes de valores fisiológicos similares en vertebrados simpátricos están bien documentados y utilizados en la literatura (Gilmore et al., 2000; Miller \& Fowler, 2015). Además, la ausencia de reportes previos de valores hormonales en sangre para el $C$. hoffmanni y otras especies de Choloepus sp., limita poder establecer comparaciones de estos valores para la especie de nuestro estudio.

El promedio de $\mathrm{P}_{4} \mathrm{H}$ obtenido en este estudio (217.46 ng/g, IC95 \%: 132.31 - 302.61 $\mathrm{ng} / \mathrm{g}$ ) es levemente mayor al reportado por Troll et al. (2013), quienes en su estudio de esteroides fecales en $C$. didactylus reportaron datos promedio para dos individuos en cautiverio $(98.0 \pm 17.0 \mathrm{ng} / \mathrm{g}$ y $105.9 \pm 30.0 \mathrm{ng} / \mathrm{g})$, variaciones que podrían estar asociadas de alguna manera con el número de muestra, o con la especificidad de detección del metabolito en el procedimiento empleado en dicho estudio. Contrario a lo esperado, los promedios de $\mathrm{E}_{2} \mathrm{H}$ obtenidos por Troll et al. (2013), fueron considerablemente más altos $(6700 \pm 900 \mathrm{pg} / \mathrm{g}$ y $7500 \pm 1600 \mathrm{pg} / \mathrm{g}$ ) en comparación con este estudio (1704.04 pg/g, IC95 \%: 1597.29 $1810.79 \mathrm{pg} / \mathrm{g})$.

Las variaciones entre las concentraciones hormonales en ambos estudios podrían deberse no sólo a las diferencias especie-específicas, 
sino también al número de deposiciones semanales, en especial por la diferencia en la recolección de heces que reporta Troll et al. (2013) en su estudio, lo cual podría ser una fuente para esta diferencia. En dicha investigación, las hembras defecaron 1.4 y 2.7 veces por semana en promedio, mientras que este estudio registró un promedio de 3.5 deposiciones por semana. Un lento tránsito intestinal podría explicar parcialmente la diferencia en los valores de $\mathrm{E}_{2} \mathrm{H}$, dado que una mayor permanencia de las heces en intestino favorecería un aumento en la concentración del metabolito.

En otras especies se ha reportado que la cantidad de fibra ingerida modifica la concentración hormonal que se excreta, de forma que la fibra dietética se asocia proporcionalmente con el $E_{2}$ (Goldin, Adlercreutz, Dwyer, Swenson, Warram, \& Gorbach, 1981; Adlercreutz et al., 1987) e inversamente con la $\mathrm{P}_{4}$ (Wasser et al., 1993). Esto sugiere que las hembras incluidas en nuestro estudio mantuvieron una dieta con menor contenido de fibra en comparación con las analizadas por Troll et al. (2013).

Asimismo, las diferencias encontradas reflejan la relevancia de evaluar las variaciones en el comportamiento hormonal a través de mediciones repetidas en un mediano plazo cuando se trabaja con muestras de heces, en vez de cuantificar un único valor (Dumonceaux et al., 2006; Schwarzenberger, 2007).

Para las cinco hembras analizadas, los valores elevados de $\mathrm{P}_{4} \mathrm{H}$ a inicios de noviembre, evidencian actividad ovárica importante, dado que los niveles de progesterona altos usualmente se asocian con la presencia de tejido luteal funcional (Reece, 2009). Sin embargo, conforme avanzó el mes de noviembre, la actividad progestacional disminuyó en frecuencia e intensidad en todas las hembras, hasta mantenerse en niveles basales en al menos tres de ellas. Esto revela una disminución de la actividad ovárica que podría estar asociada con un comportamiento reproductivo estacional, fenómeno normal relacionado con el ciclo estral reportado en otros mamíferos (Guimarães-Martins, Bonato, Queiroz-da-Silva, \& Furtado-dos-Reis, 2006). Lo anterior podría tener relación con variaciones climáticas, pues la zona en la que se ubican los animales, conocida como Caribe Sur, se caracteriza por ser muy húmeda, con dos breves periodos ligeramente menos lluviosos a lo largo del año (precipitación promedio de 100 a $200 \mathrm{~mm}$ ), y un importante incremento de las precipitaciones en noviembre y diciembre (MINAET \& IMN, 2009), con valores históricos promedio entre 400 y $445 \mathrm{~mm}$.

Si bien en el trópico, las variaciones anuales en el fotoperiodo y la temperatura ambiental son menores que en las zonas templadas, esta región presenta variaciones importantes en la precipitación y es factible que se presente cierta estacionalidad en la disponibilidad de algunos tipos de alimentos y/o el acogimiento de una estrategia reproductiva oportunista por parte de algunas especies de animales (Porras, Zarco, \& Valencia, 2004). Esto último estaría asociado con la existencia de condiciones ambientales más favorables para la supervivencia de la progenie, característica que se observa en muchas especies silvestres con el fin de garantizar una mayor tasa de supervivencia de las crías, como se reporta para otras especies de mamíferos tropicales (Guimarães-Martins et al., 2006). En Costa Rica la variación de horas luz a lo largo del año es de una hora como máximo (SuárezEsquivel \& Castro-Ramírez, 2016); aunado a lo anterior, el periodo de estudio fue de tres meses, por lo que la modificación del fotoperiodo fue aún menor y por ende, es poco probable que la influencia de horas luz haya sido determinante. Sin embargo, el primer mes del muestreo (noviembre) corresponde a la transición al periodo de mayor actividad lluviosa en el Caribe Sur de Costa Rica (MINAET \& IMN, 2009), lo cual puede ser un factor relevante en la actividad cíclica de estas hembras. De hecho, la teoría de que el ciclo reproductivo de $C$. hoffmanni ocurre con cierta estacionalidad ya ha sido propuesta (Taube et al., 2001), con un patrón de gestaciones durante la época lluviosa y partos al inicio de la época seca.

Asimismo, la presencia de fluctuaciones de $\mathrm{E}_{2} \mathrm{H}$ acompañadas de bajos niveles de $\mathrm{P}_{4} \mathrm{H}$ en las hembras (individuos tres, cuatro y cinco) 
podría deberse a ondas foliculares anovulatorias sin actividad luteal importante, fenómeno observado comúnmente en herbívoros domésticos como las yeguas (Equus caballus) (Mair, 2013) y en rumiantes domésticos (Bos sp.) en anestro posparto (Porras et al., 2004). Sin embargo, no se puede descartar la posibilidad de que esté asociado con el número de muestras o tiempo dedicado a este estudio, que pudo generar variaciones asociadas a un sesgo de muestreo.

Si bien es cierto que en todas las hembras se redujo la actividad a partir de la segunda mitad de noviembre, en las hembras uno y dos la actividad ovárica se mantuvo, aunque presentó menor intensidad

A pesar de que no se encontró una correlación estadísticamente significativa entre la $\mathrm{P}_{4}$ plasmática y la $\mathrm{P}_{4} \mathrm{H}$, los valores obtenidos en las heces del segundo día posterior a la toma de muestras de sangre reflejaron las variaciones observadas en las hormonas plasmáticas. Esta tendencia podría ser más visible con un número mayor de muestras.

Nuestros resultados sugieren que la técnica de medición de metabolitos hormonales en heces usando métodos automatizados puede resultar útil para cuantificar la actividad ovárica de los perezosos de dos dedos en cautiverio, a partir de materia fecal de individuos, aunque esto aún debe ser comprobado a través de la comparación contra otros métodos previamente verificados. Este método se podría usar para establecer relaciones fisiológicas con mayor certeza mediante la comprobación con un mayor número de muestras e individuos, además de un seguimiento más riguroso y por un periodo más prolongado.

\section{AGRADECIMIENTOS}

Este trabajo fue financiado por el Fondo Institucional para el Desarrollo Académico (FIDA) 2012 de la Universidad Nacional. Se les agradece al "Sloth Sanctuary" y a Judy Avey-Arroyo por su anuencia en colaborar y las facilidades provistas durante el muestreo; a Francisco Arroyo, Mario Romero, Nancy
Astorga y Sergio Cuadra por la asistencia técnica durante los muestreos; a Andrés Villalobos por su colaboración en el procesamiento de las muestras de hematología y química sanguínea, y al Instituto Meteorológico Nacional por la información facilitada.

\section{RESUMEN}

El uso de técnicas no invasivas ni estresantes para determinar perfiles hormonales, como la medición de esteroides fecales, ha incrementado la comprensión de la fisiología reproductiva en animales silvestres. Debido a la escasa información con respecto a perfiles hormonales reproductivos del perezoso de dos dedos, Choloepus hoffmani, se realizó un estudio en hembras en cautiverio en el centro de rescate "Sloth Sanctuary" (Cahuita, Limón, Costa Rica) con el fin de determinar (i) la confiabilidad de la extracción de progesterona y estradiol en heces, y su cuantificación en el analizador AIA-360®, (ii) evaluar los parámetros sanguíneos en esta especie y (iii) establecer si existe una correlación entre los esteroides plasmáticos y fecales. El estudio se realizó en un periodo de tres meses, durante noviembre de 2013 a enero de 2014, con un total de 208 muestras de heces provenientes de cinco hembras sexualmente maduras, con peso promedio de $6.32 \mathrm{~kg}$. El promedio de las concentraciones medianas en las heces de las cinco hembras fue $124.21 \mathrm{ng} / \mathrm{g}$ para progesterona y $1708.95 \mathrm{pg} / \mathrm{g}$ de estradiol. En plasma, los valores de mediana fueron $1.26 \mathrm{ng} / \mathrm{mL}$ con un mínimo de $0.32 \mathrm{ng} / \mathrm{mL}$ y $12.84 \mathrm{ng} / \mathrm{mL}$ como valor máximo; los valores plasmáticos de estrógeno se encontraron por debajo del límite de detección del equipo $(25 \mathrm{pg} / \mathrm{mL})$. Aunque no se encontró una correlación estadísticamente significativa entre la progesterona plasmática y la fecal, nuestros datos sugieren que los eventos plasmáticos se reflejan en heces durante los dos días posteriores. Asimismo, los niveles de progesterona se mantuvieron elevados durante la primera mitad de noviembre, y posteriormente mostraron una reducción importante en todas las hembras. Nuestros resultados demuestran que las extracciones en heces y su medición en el AIA-360 ${ }^{\circledR}$ permiten la detección y el seguimiento de variaciones hormonales en $C$. hoffmani, aunque no remplaza las mediciones plasmáticas para determinar valores absolutos.

Palabras clave: progesterona; estrógeno; heces; ciclo estral; hematología; perezosos; AIA-360.

\section{REFERENCIAS}

Adlercreutz, H., Höckerstedt, K., Bannwart, C., Bloigu, S., Hämäläinen, E., Fotsis, T., \& Ollus, A. (1987). Effect of dietary components, including lignans and phytoestrogens, on enterohepatic circulation and liver metabolism of estrogens and on sex hormone binding 
globulin (SHBG). Journal of Steroid Biochemistry, 27, 1135-1144.

Araújo, G. (2006). Aspectos clínicos e de manejo de preguiça-de-garganta-marrom Bradypus variegatus (Schinz, 1825) de vida libre na Mesorregãio Metropolitana do Recife, Pernambuco, Brasil. (Tese ao Programa de Pós-graduação). Universidade Federal Rural de Pernambuco, Pernambuco, Brasil.

Brown, J., Walker, S., \& Steinman, K. (2009). Endocrine manual for reproductive assessment of domestic and non-domestic species. Washington, United States of America: Smithsonian's National Zoological Park.

Chelini, M., Souza, N., Rocha, A., Felippe, E., \& Oliveira C. (2005). Quantification of fecal estradiol and progesterone metabolites in Syrian hamsters (Mesocricetus auratus). Brazilian Journal of Medical and Biological Research, 38, 1711-1717.

Dias, B., dos-Santos, L., Lara, P., Righetto, C., Pinder, L., \& Chiarello A. (2007). First observation on mating and reproductive seasonality in maned sloths Bradypus torquatus (Pilosa: Bradypodidae). Journal of Ethology, 27, 97-103.

Dumonceaux, G., Bauman, J., \& Carillo G. (2006). Evaluation of progesterone levels in feces of captive reticulated giraffe (Giraffa camelopardalis reticulata). Journal of Zoo and Wildlife Medicine, 37, 255-261.

Durán, A. 2005. Valores de hematología y de bioquímica sanguínea del perezoso de tres dedos (Bradypus variegatus) y el perezoso de dos dedos (Choloepus hoffmanni) en cautiverio en Limón, Costa Rica. (Tesis de Licenciatura). Universidad Nacional, Heredia, Costa Rica.

Gilmore, D., Da-Costa, D., \& Duarte, D. (2000). An update on the physiology of two- and three-toed sloths. Brazilian Journal of Medical and Biological Research, 33, 129-146.

Goldin, B. R., Adlercreutz, H., Dwyer, J. T., Swenson, L., Warram, J. H., \& Gorbach, S. L. (1981). Effect of Diet on Excretion of Estrogens in Pre and Postmenopausal Women. Cancer Research, 41, 3771 -3773.

Goymann, W. (2005). Noninvasive monitoring of hormones in bird droppings: physiological validation, sampling, extraction, sex differences, and the influence of diet on hormone metabolite levels. Annals of the New York Academy of Sciences, 1046, 35-53.

Goymann, W. (2012). Review: On the use of non-invasive hormone research in uncontrolled, natural environments: the problem with sex, diet, metabolic rate and the individual. Methods in Ecology and Evolution, $3,757-765$.

Graham, L. (2004). Non-invasive monitoring of reproduction in zoo and wildlife species. Annual Review of Biomedical Sciences, 6, 91-98.
Graham, L., Schwarzenberger, F., Möstl, E., Galama, W., \& Savage, A. (2001). A versatile enzyme immunoassay for the determination of progestogens in feces and serum. Zoo Biology, 20, 227-236.

Guimarães-Martins, E., Bonato, V., Queiroz-da-Silva, C., \& Furtado-dos-Reis, S. (2006). Seasonality in reproduction, age structure and density of the gracile mouse opossum Gracilinanus microtarsus (Marsupialia: Didelphidae) in a Brazilian cerrado. Journal of Tropical Ecology, 22, 461-468.

Hanley, C. (2008). Immobilization of free-ranging Hoffmann's two-toed (Choloepus hoffmanni) and brown-throated three-toed (Bradypus variegatus) sloths using ketamine and medetomidine: a comparison of physiologic parameters. Journal of Wildlife Diseases, 44, 938-945.

Hayssen, V. (2011). Choloepus hoffmanni (Pilosa: Megalonychidae). Mammalian Species, 43, 37-55.

Keay, J., Singh, J., Gaunt, M., \& Kaur, T. (2006). Fecal glucocorticoids and their metabolites as indicators of stress in various mammalian species: a literature review. Journal of Zoo and Wildlife Medicine, 37, 234-244.

Kinney, M., Cole, G., Vaughan, C., \& Sladky, K. (2013). Physiologic and serum biochemistry values in freeranging hoffmann's two-toed (Choloepus hoffmanni) and brown-throated three-toed (Bradypus variegatus) sloths immobilized using dexmedetomidine and ketamine. Journal of Zoo and Wildlife Medicine, 44, 570-580.

Mair, T. S. (2013). Equine medicine, surgery and reproduction. London, England: W.B. Saunders.

Meneses-Guevara, A., \& Bouza-Mora, L. S. (2014). Manual de hematología y química clínica en medicina veterinaria. Heredia, Costa Rica: EUNA.

Miller, R. E., \& Fowler, M E. (2015). Fowler's Zoo and Wild Animal Medicine. Volume 8. St Louis, Missouri: Elsevier Saunders.

MINAET (Ministerio de Ambiente, Energía y Telecomunicaciones) \& IMN (Instituto Meteorológico Nacional de Costa Rica). (2009). Segunda Comunicación Nacional a la Convención Marco de la Naciones Unidas sobre Cambio Climático. San José, Costa Rica.

Mühlbauer, M., Duarte, D., Gilmore, D. P., \& Da-Costa, C. (2006). Fecal estradiol and progesterone metabolite levels in the three-toed sloth (Bradypus variegatus). Brazilian Journal of Medical and Biological Research, 39, 289-295.

Palme, R. (2005). Measuring fecal steroids: guidelines for practical applications. Annals of the New York Academy of Sciences, 1046, 75-80. 
Paris, M., Whiteb, A., Reissb, A., Westa, M., \& Schwarzenberger F. (2002). Faecal progesterone metabolites and behavioural observations for the non-invasive assessment of oestrous cycles in the common wombat (Vombatus ursinus) and the southern hairy-nosed wombat (Lasiorhinus latifrons). Animal Reproduction Science, 72, 245-257.

Pereira, R., Barbanti, J., \& Negrão J. (2005). Seasonal changes in fecal testosterone concentrations and their relationship to the reproductive behavior, antler cycle and grouping patterns in free-ranging male Pampas deer (Ozotoceros bezoarticus bezoarticus). Theriogenology, 63, 2113-2125.

Porras, A., Zarco, L., \& Valencia, J. (2004). Estacionalidad reproductiva en ovejas. Ciencia Veterinaria, 9, 1-33.

R Core Team. (2014). R: A language and environment for statistical computing. $R$ Foundation for Statistical Computing (versión 3.3.1) [Software]. Vienna, Austria: The R Project for Statistical Computing. Recuperado de http://www.R-project.org/ (Consulta: 25 marzo, 2015).

Reece, W. O. (Ed). (2009). Dukes fisiología de los animales domésticos. 1.ed. Zaragoza, España: Acribia.

Schwarzenberger, F. (2007). The many uses of noninvasive faecal steroid monitoring in zoo and wildlife species. International Zoo Yearbook, 41, 52-74.

Sherman, R. A. (2015). Multicon: Multivariate Constructs. $R$ package (versión 1.6) [Software]. Vienna, Austria: The R Project for Statistical Computing. Recuperado de http://CRAN.R-project.org/package=multicon (Consulta: 7 de julio, 2017).

Signorell, A., Aho, K., Alfons, A., Anderegg, N., Aragon, T., ... Zeileis. A. (2017). DescTools: Tools for descriptive statistics. $R$ package (versión 0.99.21) [Software]. Vienna, Austria: The R Project for Statistical Computing. Recuperado de http://CRAN.R-project. org $/$ package $=$ Desc Tools

Snoeck, P. P. N., Cruz, A. C. B., Catenacci, L. S., \& Cassano, C. R. (2011). Citologia vaginal de preguiça-decoleira (Bradypus torquatus). Pesquisa Veterinária Brasileira, 31, 271-275.
Suárez-Esquivel, M., \& Castro-Ramírez, L. (2016). Measurement of thyroid hormones and cortisol in horses with an automated immunoassay analyzer. Revista Ciencias Veterinarias, 34(1), 39-49. doi: 10.15359/ rcv.34-1.3

Taube, E., Keravec, J., Vié, J., \& Duplantier, J. (2001). Reproductive biology and postnatal development in sloths, Bradypus and Choloepus: review with original data form the field (French Guiana) and from captivity. Mammal Review, 31, 173-188.

Touma, C., \& Palme, R. (2005). Measuring fecal glucocorticoid metabolites in mammals and birds: the importance of validation. Annals of the New York Academy of Sciences, 1046, 54-74.

Troll, S., Gottschalk, J., Seeburger, J., Ziemssen, E., Häfner, M., Thielebein, J., \& Einspanier, A. (2013). Characterization of the ovarian cycle in the two-toed sloths (Choloepus didactylus): an innovative, reliable, and noninvasive method using fecal hormone analyses. Theriogenology, 80, 275-283.

Valdespino, C., Martínez-Mota, R., García-Feria, L. M., \& Martínez-Romero L. E. (2007). Evaluación de eventos reproductivos y estrés fisiológico en vertebrados silvestres a partir de sus excretas: evolución de una metodología no invasiva. Acta Zoológica Mexicana, $23,151-180$.

Vogel, I., Vie, J., Thoisy, B., \& Moreau B. (1999). Hematological and serum chemistry profiles of free-ranging southern two-toed sloths in French Guiana. Journal of Wildlife Diseases, 35, 531-535.

Wainwright, M. (2007). The mammals of Costa Rica: a natural history and field guide. 1.ed. New York, USA: Comstock Publishing Associates.

Wasser, S. K., Thomas, R., Nair, P. P., Guidry, C., Southers, J., Lucas, J., Wildt, D. E., \& Monfort S. L. (1993). Effects of dietary fibre on faecal steroid measurements in baboons (Papio cynocephalus cynocephalus). Journal of Reproduction and Fertility, 97, 569-574.

Wickham, H. (2009). Ggplot2: elegant graphics for data analysis. New York, USA: Springer. 